\title{
Resolver Emulation for PMSMs Using Low Cost Hall Effect Sensors
}

\author{
Daniel Fernandez \\ University of Oviedo. Dept. of Elect., \\ Computer \& System Engineering, \\ Gijón, 33204, Spain \\ fernandezalodaniel@,uniovi.es \\ David Reigosa \\ University of Oviedo. Dept. of Elect., \\ Computer \& System Engineering, \\ Gijón, 33204, Spain \\ diazdavid@uniovi.es
}

\author{
Diego Fernandez \\ University of Oviedo. Dept. of Elect., \\ Computer \& System Engineering, \\ Gijón, 33204, Spain \\ dflaborda@uniovi.es \\ Alberto B. Diez \\ University of Oviedo. Dept. of Elect., \\ Computer \& System Engineering, \\ Gijón, 33204, Spain \\ abdiez@uniovi.es
}

\author{
Maria Martinez \\ University of Oviedo. Dept. of Elect., \\ Computer \& System Engineering, \\ Gijón, 33204, Spain \\ martinezgmaria@uniovi.es \\ Fernando Briz \\ University of Oviedo. Dept. of Elect., \\ Computer \& System Engineering, \\ Gijón, 33204, Spain \\ fernando@isa.uniovi.es
}

\begin{abstract}
Control of permanent magnet synchronous machines (PMSMs) requires absolute rotor position measurement/estimation, as well as the magnet polarity detection for the machine start-up, encoders/resolvers being normally used for this purpose. However, these sensors can account for a large portion of the overall drive cost, and require additional room and cabling, therefore penalizing the size and reliability of the drive. This paper proposes a method to emulate a resolver in machines using low cost Hall-effect sensors. The proposed Hall-effect resolver (HER) system is a new type of angular position sensor for PMSMs. This allows to control machines which do not include a resolver from inverters which require a resolver signal to operate.
\end{abstract}

Keywords-Permanent magnet synchronous machines, resolver emulation, rotor speed \& position measurement, Hall effect sensors

\section{Introduction}

Permanent magnet synchronous machines (PMSMs) have become very popular during the last decades due to their high efficiency, high power density and superior dynamic response compared with other types of machines, e.g. induction, wound field or synchronous reluctance machines. Control of PMSMs requires the absolute position of the rotor, i.e. including magnet polarity prior to startup of the machine [1]-[2]. Both absolute encoders [3]-[6] and resolvers [16]-[22] comply with this requirement. On the contrary, incremental encoders will require some additional mechanism to obtain the rotor initial position. Combined use of incremental encoder and Hall-effect sensors [3]-[10] provide the absolute position,.

\footnotetext{
1 This work was supported in part by the Research, Technological Development and Innovation Programs of the Spanish Ministry Economy and Competitiveness, under grant MINECO-17-ENE2016-80047-R and by the Government of Asturias under project IDI/2018/000188 and FEDER funds
}

Table I shows a comparative analysis of position sensors normally used in industry applications. Optical encoders are likely the preferred option in general purpose applications; however, they are less robust than magnetic encoders and resolvers, the last being the preferred option for e.g. hybridelectric vehicles (HEV) and electric vehicles (EV) motors [13][15], aircrafts, satellite antennas or robots [11]-[13]. Although resolvers provide absolute position, its use always implies additional hardware (a resolver to digital converter is needed [12],[17]-[18]), room and cabling, therefore penalizing cost, size and reliability of the drive.

In addition of conventional position sensors, a number of alternative solutions have been reported in the literature. A planar angular position sensor is proposed in [23], with a similar working principle as a variable reluctance resolver. However, this sensor is highly sensitive to electromagnetic interference due to its construction and requires and additional electronic circuit to operate. The sensor proposed in [24] provides angular

\begin{tabular}{|c|c|c|c|c|}
\hline \multicolumn{5}{|c|}{ Table I. Position sensors } \\
\hline & Cost & Accuracy & Robustness & Position \\
\hline $\begin{array}{|ll|}\text { Incremental optical } \\
\text { encoder [3]-[6] }\end{array}$ & $\uparrow$ & 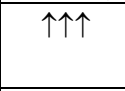 & $\downarrow$ & Incremental \\
\hline $\begin{array}{l}\text { Incremental magnetic } \\
\text { encoder[8]-[10] }\end{array}$ & $\uparrow$ & $\uparrow$ & $\uparrow$ & Incremental \\
\hline $\begin{array}{l}\text { Absolute optical encoder } \\
{[3]-[6]}\end{array}$ & $\uparrow \uparrow \uparrow$ & $\overline{c \uparrow \uparrow \uparrow}$ & $\downarrow$ & Absolute \\
\hline \begin{tabular}{|ll}
$\begin{array}{l}\text { Wound field resolver } \\
{[16]-[19]}\end{array}$ & \\
\end{tabular} & $\overline{\uparrow \uparrow \uparrow \uparrow}$ & $\uparrow \uparrow \uparrow$ & $\overline{\uparrow \uparrow}$ & Absolute \\
\hline $\begin{array}{l}\text { Variable reluctance } \\
\text { resolver [20]-[21] }\end{array}$ & $\uparrow$ & $\uparrow \uparrow$ & $\uparrow \uparrow \uparrow \uparrow$ & Absolute \\
\hline \begin{tabular}{|ll} 
Planar & Coil/Capacitor \\
{$[23]-[25]$} & \\
\end{tabular} & ND & $\uparrow$ & $\downarrow \downarrow$ & Absolute \\
\hline $\begin{array}{|ll|}\text { Contactless } & \text { Hall-based } \\
{[26]-[27]} & \\
\end{array}$ & $\downarrow \downarrow \downarrow$ & $\downarrow$ & $\uparrow$ & Absolute \\
\hline Proposed HER & $\downarrow \downarrow \downarrow$ & $\uparrow$ & $\uparrow \uparrow$ & Absolute \\
\hline
\end{tabular}


position output that is not compatible with standard encoder or resolver signals. In [25], a contactless capacitive angular position sensor is proposed, but it is characterized for presenting a non-linear behavior. Other non-contact angular positions sensors based on Hall-effect devices for automotive applications (e.g. throttle position detection, shaft position...) are also commercially available [26], [27]. The main drawbacks of these methods are the robustness decrease, inertia increase and extra room.

This paper proposes a method to emulate resolvers using the signals provided by low-cost Hall-effect sensors, HER, which is an extension of [28]. Stability of the HER system is evaluated and compared with standard sensors. Uses of the HER system could be twofold: 1) it would enable the use of machines that do not include a resolver, with electric drives that require a resolver signal to operate and 2) a new type of position sensor for PMSM. Appealing properties of the HER system are reduced cost as well as simple, light and robust construction, as there are no moving parts or couplings.

The paper is organized as follows: design and operation principles of resolvers are presented in section II, emulation of a resolver using Hall-effect sensors is presented in section III, machine and Hall-effect sensors placement is presented in section IV, experimental results are presented in section $\mathrm{V}$ and conclusions are presented in section VI.

\section{Resolver design and principles of operation}

This section briefly reviews the design and operating principles of resolvers. Commercial resolvers can be classified into wound field (WF) [16]-[19] and variable reluctance (VR) [20]-[21]. WF resolvers include an excitation winding in the rotor, which is magnetically coupled with two identical stator windings having $90^{\circ}$ mutual electrical phase shift [16]-[19]. A sinusoidal voltage, typically called carrier, is applied to the rotor windings, via brushes and rings or alternatively by means of a magnetic coupling (i.e. rotating transformer) [16]-[19]. The resulting voltages in the two-stator windings are a sine and cosine signal whose angle is modulated by the rotor angle. WF resolvers have a wide temperature range of operation, are small and light, and with a simple and robust construction; in addition they are highly insensitive to noise and tolerate long transmission cables length [16]-[19]. On the other hand, VR resolvers have both the output and the excitation windings in the stator; no brushes or rotating transformer being therefore required. In general, VR resolvers are even less expensive and more robust than WF resolvers [13]-[14], [20]-[21]. a)

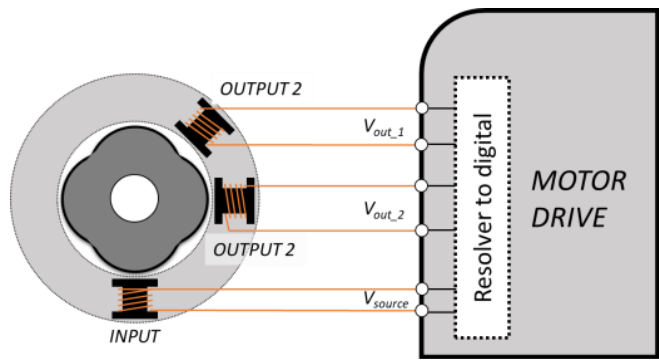

b)
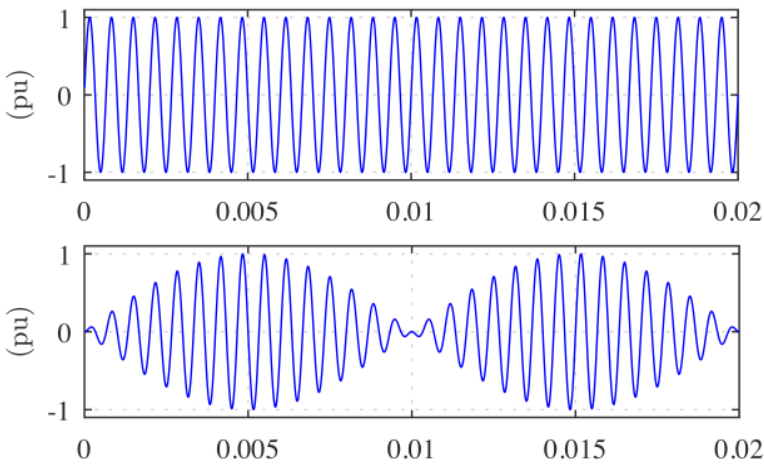

c)

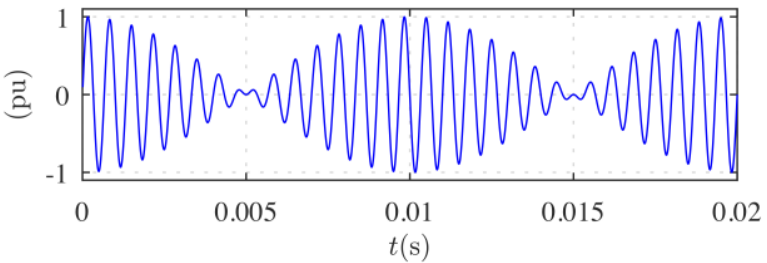

d)

Fig. 1.-. Basic operation of a VR resolver. a) Schematic representation of VR resolver and motor drive, b) excitation of the resolver, $V_{\text {source }}$, c) output 1 of the resolver, $V_{\text {out_l }}$ (i.e. sine), d) output 2 of the resolver, $V_{\text {out_2 }}$ (i.e. cosine). $\omega_{\text {source }}=2 \cdot \pi \cdot 500 \mathrm{rad} / \mathrm{s}, \omega_{\mathrm{r}}=2 \cdot \pi \cdot 50 \mathrm{rad} / \mathrm{s}$.

Resolvers can be seen as a special type of rotary transformer that couples a primary winding (Input, see Fig. 1a) with two secondary windings (Output 1 \& 2, Fig. 1a) that are 90 electrical degrees phase shifted; note that Fig. 1a shows a schematic representation of a VR resolver. Excitation signal, i.e. $V_{\text {source }}$ in Fig. 1a, is typically a sinusoidal signal (1), see Fig. 1b, where $V_{0}$ and $\omega_{\text {source }}$ are the magnitude and frequency of the excitation signal respectively. The excitation frequency depends on the resolver type. Brushless resolvers typically operate in the range of 1 to $10 \mathrm{kHz}$, while Variable Reluctance resolvers operate around $10 \mathrm{kHz}$. For the sake of compatibility with conventional resolvers, frequencies of $2 \mathrm{kHz}$ and $10 \mathrm{kHz}$ will be used in this paper.

The output signals of the resolver are (2) and (3), $V_{\text {out }} l$ and $V_{\text {out_2 }}$ respectively, see Fig.1c and Fig.1d, where $k$ is the transformation ratio of the resolver, $\theta_{r}$ is the position of the resolver, $\omega_{r}$ is the rotating frequency of the resolver and $X$ is a multiplication factor for the angle [13].

$$
\begin{aligned}
& V_{\text {source }}=V_{0} \sin \left(\omega_{\text {source }} t\right) \\
& V_{\text {out_ } 1}=V_{0} \sin \left(\omega_{\text {source }} t\right) * k * \sin \left(X \theta_{r}\right) \\
& V_{\text {out_ } 2}=V_{0} \sin \left(\omega_{\text {source }} t\right) * k * \cos \left(X \theta_{r}\right)
\end{aligned}
$$


$V_{\text {out_l }}$ and $V_{\text {out_2 }}$ fed a resolver-to-digital (R/D) converter [11]-[22], which typically includes a demodulation stage [12], [17]-[19], [32], [33]; $V_{\text {out_l }}$ and $V_{\text {out_2 }}$ after the demodulation being expressed by (4) and (5); A large variety of methods have been proposed to obtain the rotor position, $\theta_{r}$, from (4) and (5) [12], [17]-[19], [32], [33].

$$
\begin{aligned}
& V_{\text {out }_{-1}^{\prime}}^{\prime}=k * \sin \left(X \theta_{r}\right) \\
& V_{\text {out_ } 2}^{\prime}=k * \cos \left(X \theta_{r}\right)
\end{aligned}
$$

\section{Emulation of a resolver using Hall-effect sensors}

This section presents the proposed system to emulate a resolver using Hall-effect sensors.

Hall-effect sensors are typically used to convert a magnetic flux density signal into a voltage signal (i.e. Hall voltage). It consists on a semiconductor element (i.e. Hall element), see Fig. 2, which fed with a current $I_{z}$. In the presence of a magnetic field $\left(\overline{B_{y}}\right.$ in Fig. 2), the induced Hall voltage $\left(\mathrm{V}_{\mathrm{H}}\right)$ is proportional to both the current applied, $I_{z}$, and the magnetic flux density, $\overline{B_{y}}$, (6), where $h w$ is the cross-sectional area of the element, $e$ is the charge of the electron and $n$ stands for the charge carrier density. Since $e, h w$ and $n$ are constants, $\mathrm{V}_{\mathrm{H}}$, will be proportional to $\overline{B_{y}}$ and $\mathrm{I}_{\mathrm{z}}$.

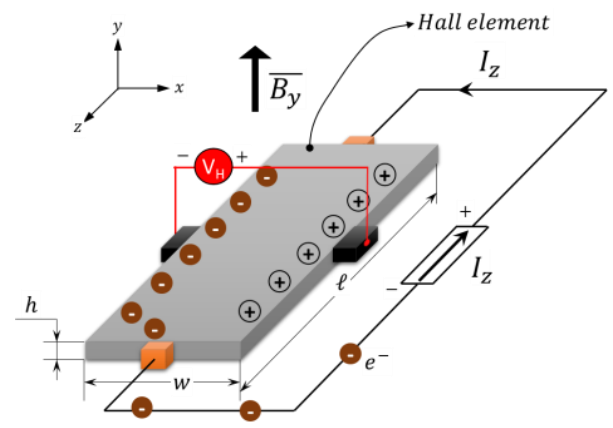

Fig. 2. Schematic representation of the Hall-effect phenomenon

$V_{H}=\frac{I_{Z} \bar{B}_{y}}{n h w e}$

$V_{H}=k\left(I_{Z} \bar{B}_{y}\right)$

Fig. 3 shows and schematic representation of the test machine that will be used for the experimental verification of the proposed HER system. The HER system consists of two Hall-effect sensors, 90 electrical degrees phase shifted, HALL1 and HALL-2 in Fig. 3a. The sensors will be mounted on the end frame of the machine, measuring field in the radial direction; i.e. the sensors will measure the leakage flux in y-axis direction $\left(\mathrm{B}_{\mathrm{y}}\right)$, see Figs. $3 \mathrm{~b}$ and $\mathrm{c}$, which will vary as the rotor rotates [29]. The output of HALL-1 and HALL-2 will consist of a sine and cosine waveforms containing the position information [28]-[30].

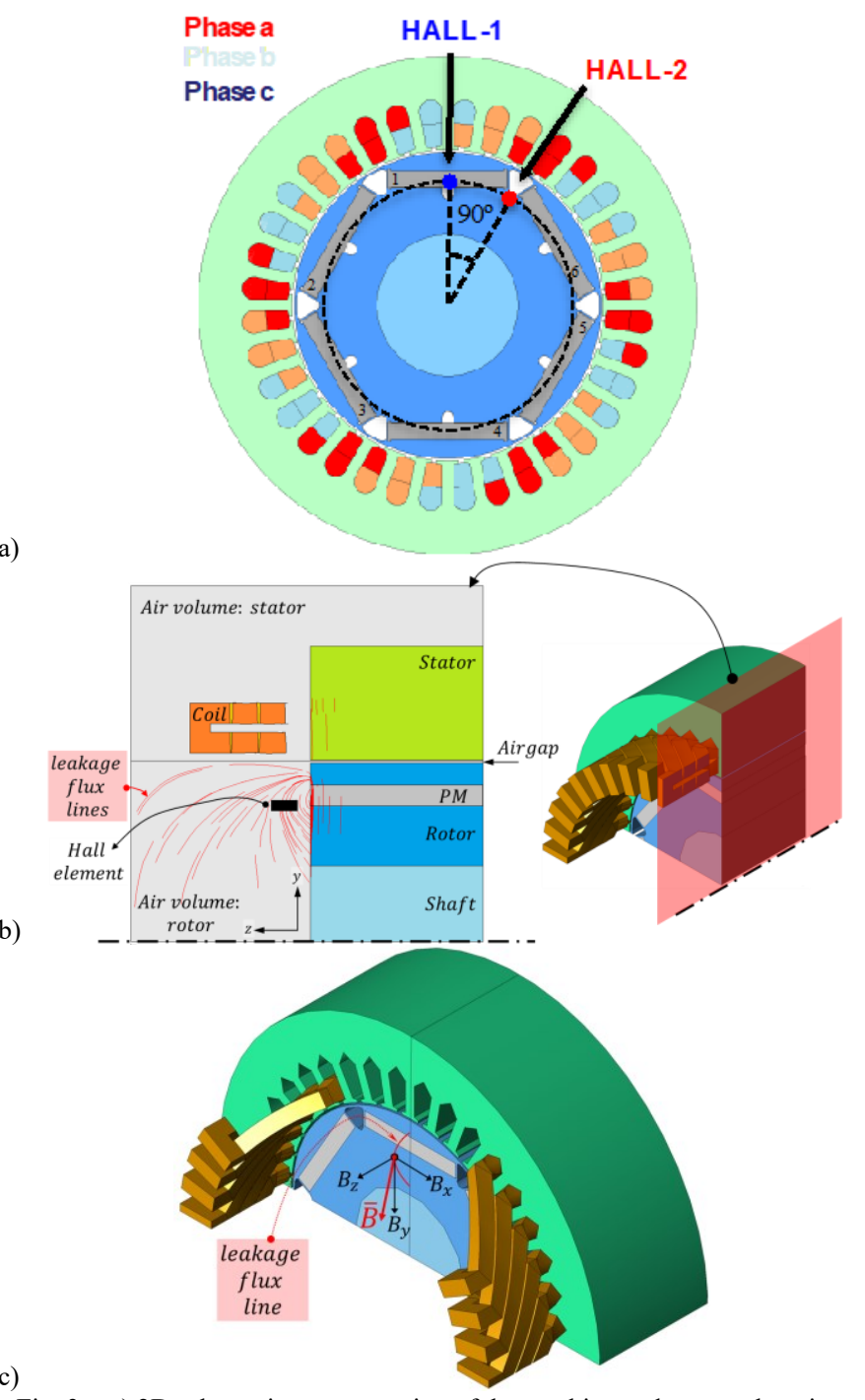

Fig. 3.-. a) 2D schematic representation of the machine and sensors location on the cross section (x-y), b) FEA result of a leakage flux and sensor location, $y-z$ plane, and c) sensor location and leakage flux vector components.

Hall sensors are normally fed using a DC current. The resulting output signals of the two Hall-effect sensors are shifted by $90^{\circ}$ as shown in Fig. 4 a.

It is possible however, to feed the sensors using a sinusoidal signal (either voltage or current), i.e. same as WF and VR resolvers [16]-[21]. The output signals from the two Hall-effect sensors are shown in Fig. 4b. The peak value of the resulting signals will be proportional to the magnet leakage flux density seen by the sensor and to the current feeding the sensor (7). Similarities between the signals in Fig. $4 \mathrm{~b}$ are those produced by traditional resolvers in Fig.1c-d evident, what strongly support the idea that the same signal processing used with the resolver signals could be applied to the signals produced by the hall sensors. It is noted that in this case, there is no element 
attached to the rotor, the solution being therefore simple, compact and robust.
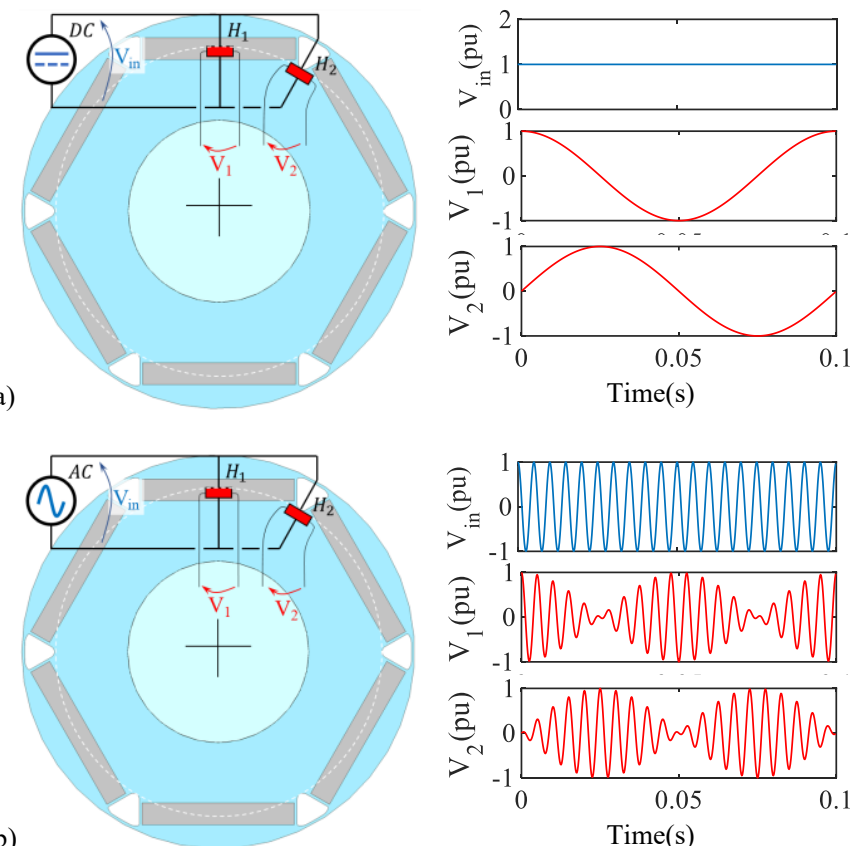

Time(s)

Fig. 4.-. a) Schematic representation of the Hall-effect sensors supply system and resulting signals as the machine rotates at $\omega=10 \mathrm{~Hz}$ for: a) DC excitation and b) AC excitation.

It must be noted that the proposed method assumes a linear response of the sensor with the excitation signal. To validate this assumption, a sensor was placed on top of a PM (see Fig. $5 \mathrm{a}$ ), being subjected therefore to a constant field. The sensor was fed with a sinusoidal voltage $V_{i n}$ shown in Fig. $5 \mathrm{~b}$ (see Fig. $5 \mathrm{a})$. Fig. $5 \mathrm{c}$ and $\mathrm{d}$ show the output of the sensor, $V_{\text {out }}$, and excitation signal $V_{i n}$ vs. output of the sensor $\left(V_{i n}-V_{\text {out }}\right.$, see Fig. $5 d)$. The results in Fig. 5 confirm the linear response of the sensor with respect its sinusoidal excitation.

a)

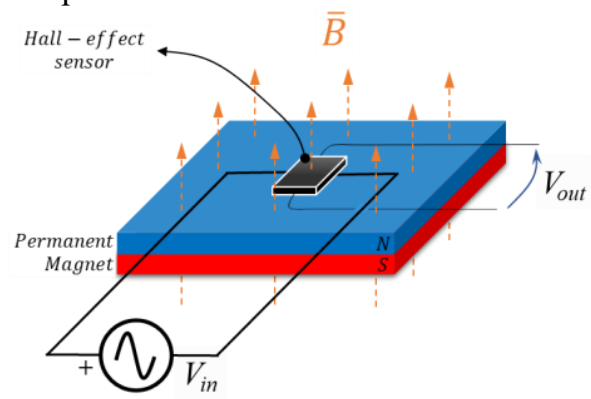

b)

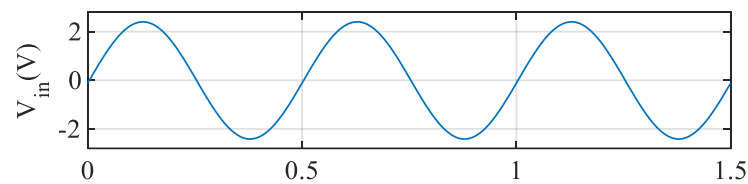

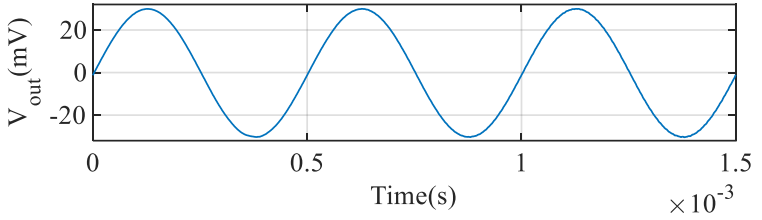

c)

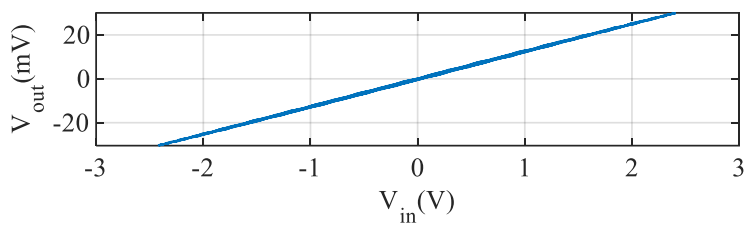

Fig. 5. a) schematic representation of the setup for testing Hall effect sensor linearity, b) excitation of the Hall-effect sensor, $V_{i n}$, c) output of the Halleffect sensor, $V_{\text {out }}$, and e) $V_{\text {source }}$ vs. $V_{\text {out }}$. $\omega_{\text {source }}=2 \cdot \pi \cdot 2000 \mathrm{rad} / \mathrm{s}, V_{\text {source }}=$ $2.3 \mathrm{~V}$.

\section{Experimental test bench}

The HER system has been implemented in an IPMSM, its design being shown in Fig.3; Table II shows the ratings and dimensions of the machine. Hall-effect sensors [31], have been attached to the end shield to the machine, see Fig. c; i.e. the sensors are placed between the rotor and end shield.

Table II. Machine parameters

\begin{tabular}{|c|c|c|c|}
\hline$P_{\text {RATED }}(\mathrm{kW})$ & 7.5 & $I_{\text {RATED }}(\mathrm{A})$ & 14 \\
\hline$\omega_{\text {RATED }}(\mathrm{rpm})$ & 1000 & Stator slots & 36 \\
\hline Poles & 6 & Rotor radius (mm) & 54.2 \\
\hline $\begin{array}{l}\text { Magnet dimensions: width, } \\
\text { height and length (mm) }\end{array}$ & $42 \times 6 \times 10$ & $\begin{array}{l}\text { Magnet position from shaft } \\
(\mathrm{mm})\end{array}$ & 44.6 \\
\hline Airgap length (mm) & 0.8 & Inner stator radius (mm) & 55 \\
\hline Outer stator radius (mm) & 88 & \begin{tabular}{|l} 
Sensor position: \\
Radius $(\mathrm{mm})$ \\
Distance from the rotor in \\
axial direction $(\mathrm{mm})$
\end{tabular} & $\begin{array}{c}44.6 \\
5\end{array}$ \\
\hline
\end{tabular}

Assembly of the sensors does not differ from standard Halleffect sensors already available in some machines [34]-[35], while cabling between sensors and drive is the same as for standard resolvers [16]-[21]. The phase shift among sensors is 90 electrical degrees, which corresponds to 30 mechanical degrees in a 6-pole machine, see Fig. 6a; the output of the two Hall-effect sensors will be therefore two sinusoidal signals, $90^{\circ}$ phase shifted (i.e. sine and cosine waveforms). Hall-effect sensors that have been used, [31], are standard sensors typically used for motion control in PMSMs [28]-[30]. Standard Halleffect sensors used in PMSM drives measure the magnetic flux density along one direction, i.e. they are 1D sensors; Hall-effect sensors used in this work are oriented in the radial direction, i.e. $\mathrm{y}$-axis direction (see Fig.6a), which is expected to provide high sensitivity to PM leakage flux and low sensitivity to stator current [28]-[30]. 


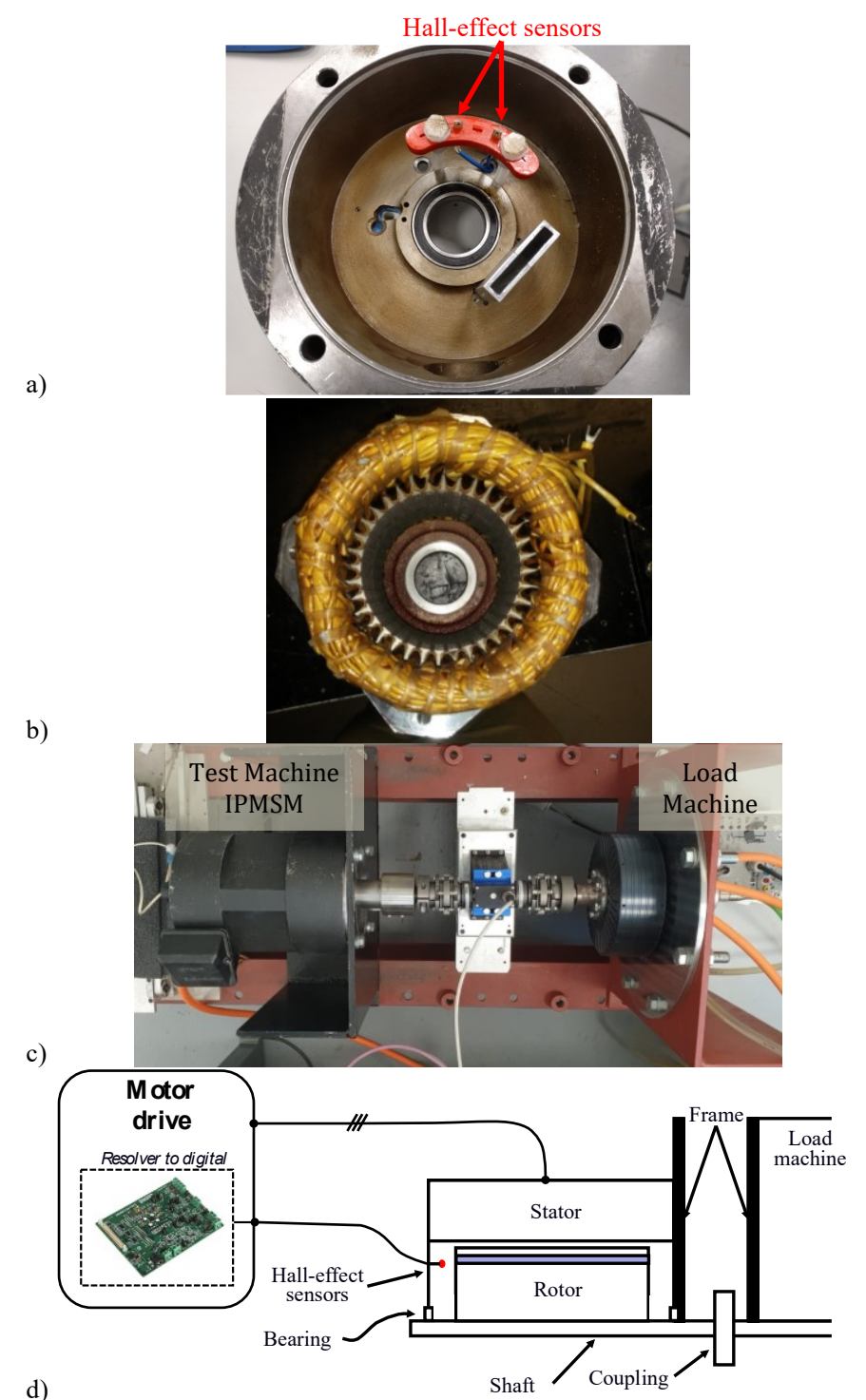

Fig. 6.-. Machine desing and experimental setup. a) machine end shield with the Hall-effect sensors, b) machine stator, c) test bench and d) schematic representation of the test bench and drive.

Stator of the machine is shown in Fig. 6b. Fig. 6c shows the test bench consisting of the test machine and a load machine [36]. Fig. 6d shows a schematic representation of the test bench, including load machine, test machine and connection between the test machine and drive. It can be observed from Fig. $6 \mathrm{~d}$ that the Hall-effect sensors are mounted on the end shield of the nondrive end of the machine; Hall-effect sensors being connected to the "Resolver to digital" control board of the drive [37].

\section{Experimental results}

Experimental results of the HER system are presented in this section for different working conditions of the machine.

A $2 \mathrm{kHz}, 5 \mathrm{~V}_{\text {peak }}$ signal was used to feed the sensor in Figs. 7$8,10 \mathrm{kHz} 10 \mathrm{~V}$ was used in Figs 10 to 13 . These signals are similar to those used by conventional resolvers, meaning that they can feed directly any existing circuitry designed for the signal processing of conventional resolvers.
It is finally noted that the input impedance of the Hall sensor is $\sim 725 \Omega$ [31], the total power consumption of the HER sensor (RMS) being $\sim 34.5 \mathrm{~mW}(\sim 17.2 \mathrm{~mW}$ per sensor). Input impedance of conventional resolvers (VR and Brushless) varies from $\sim 50 \Omega$ to $\sim 150 \Omega$, leading to power consumptions in the range of 250 to $500 \mathrm{~mW}$.

a)

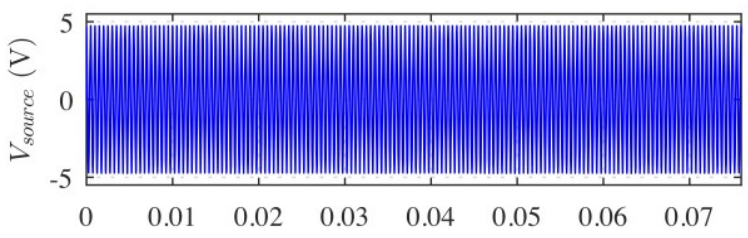

b)
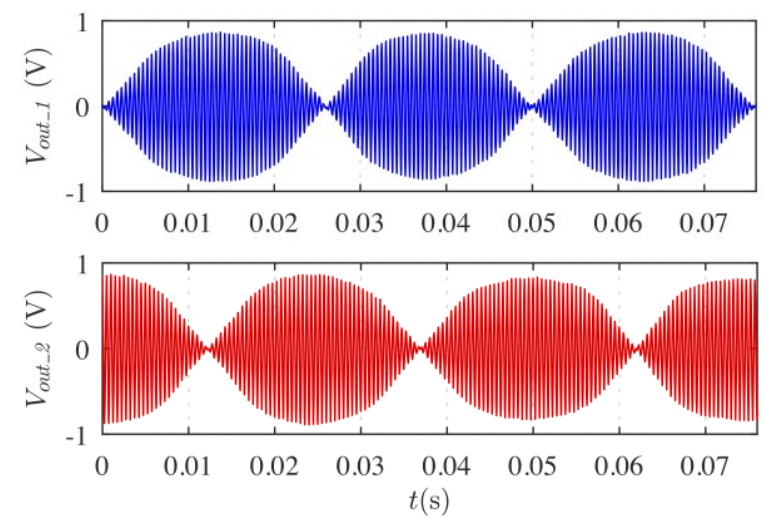

Fig. 7.-. a) Excitation of the Hall-effect sensors, $V_{\text {source }}$ b) output of Halleffect sensor 1, $V_{\text {out } I}$ (see Fig. ), and c) output of Hall-effect sensor 2, $V_{\text {out } 2}$ (see Fig. ). $\omega_{\text {source }}=2 \cdot \pi \cdot 2000 \mathrm{rad} / \mathrm{s}, V_{\text {source }}=5 \mathrm{~V}, \omega_{r}=2 \cdot \pi \cdot 40 \mathrm{rad} / \mathrm{s}, I_{d}=I_{q}$ $=0$ pu.
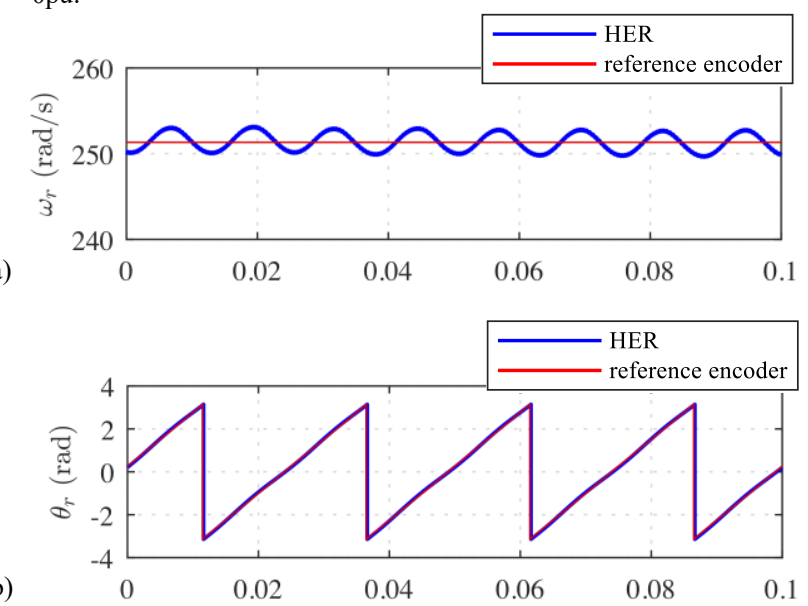

b)

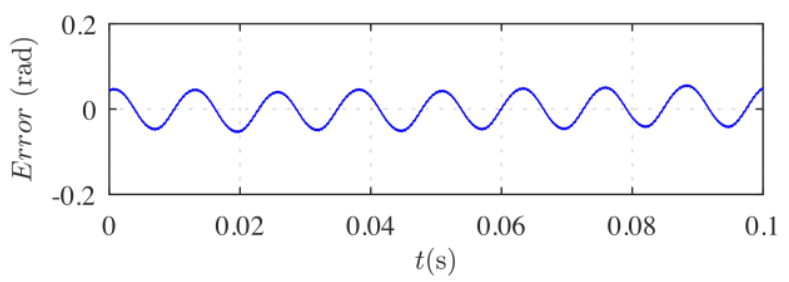

Fig. 8.-. Experimental results using the HER system (blue) and a conventional encoder (red); a) measured speed, b) measured position and c) position error. $\omega_{\text {source }}=2 \cdot \pi \cdot 2000 \mathrm{rad} / \mathrm{s}, V_{\text {source }}=5 \mathrm{~V}, \omega_{r}=2 \cdot \pi \cdot 40 \mathrm{rad} / \mathrm{s}, I_{d}$ $=I_{q}=0 \mathrm{pu}$. 


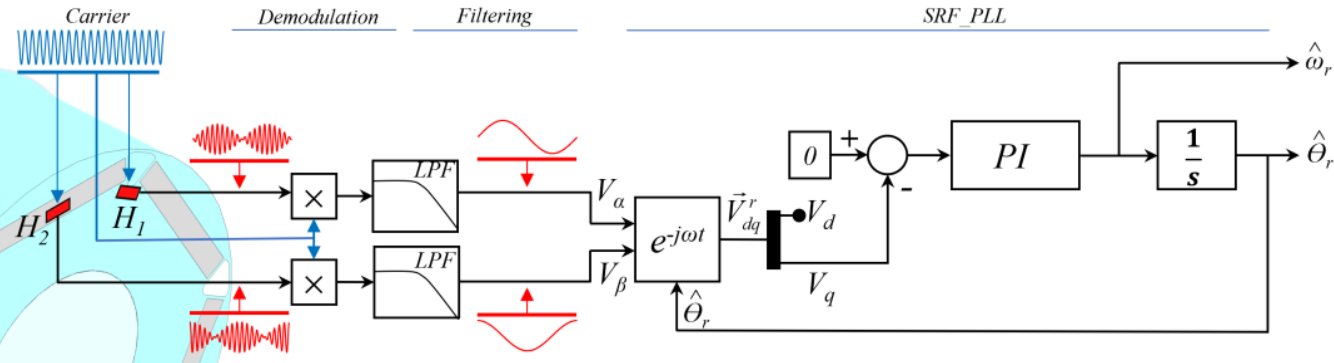

Fig. 9. Schematic representation of the signal processing used for speed and position estimation.

Fig. $7 \mathrm{~b}$ and $\mathrm{c}$ show the output signals of the two Hall-effect sensors, $V_{\text {out_l }}$ (sine) and $V_{\text {out_2 }}$ (cosine) when the machine is rotating at $40 \mathrm{~Hz}$ and with no fundamental current. Fig. $8 \mathrm{a}$ and $8 \mathrm{~b}$ shows the speed and position measured using the HER sensor, an encoder with 4096 pulses per revolution is also shown for comparison purposes. Figs. 8c shows position error. It is observed that under no load condition the peak error in the estimated position is $<0.07 \mathrm{rad}$.

Fig. 9 shows the signal processing used for speed and position estimation. Measured flux densities $H_{1}$ and $H_{2}$ are multiplied by the carrier signal at the demodulation. A second order low pass filter with a cut-off frequency of $1 \mathrm{kHz}$ is used to remove high frequency noise. The angle of the resulting $V_{\alpha}$ and $V_{\beta}$ signals (9) is obtained using a synchronous reference frame phase-locked loop (SRF-PLL), which provides the estimated rotor speed and position [38].

$\vec{V}_{\alpha \beta}^{s}=V_{\alpha}+j V_{\beta}$

$\vec{V}_{d q}^{r}=\vec{V}_{\alpha \beta}^{s} e^{-j \theta_{r}}$

The bandwidth of the proposed HER will depend primarily on the bandwidth of the PLL, which has been set to $230 \mathrm{~Hz}$. The bandwidth of the Hall sensor $250 \mathrm{kHz}$, and can be therefore safely neglected [31].

A. Influence of dq-axes currents

Both $d$ - and $q$ - axes stator currents [29] are expected to interfere with the leakage flux due to the magnets measured by the Hall effect sensors. $d$-axis current will affect only to the amplitude of the measured signals, but with no effect on the angles, as it is aligned with the magnet. $q$-axis current affects the phase of the leakage flux waveform, resulting therefore in a position error.

Fig. 10a shows the estimated position error when $d$-axis current is varied from 0 to $-1 \mathrm{pu}$ (i.e. flux weakening) in steps of $0.25 \mathrm{pu}$. As expected, $d$-axis currents do not affect to the accuracy. Similarly Fig $9 \mathrm{~b}$ shows the results when $q$-axis current is varied from 0 to $1 \mathrm{pu}$ in steps of $0.25 \mathrm{pu}$. As expected, the error increases almost linearly with the $q$-axis current. However, this error can be compensated, two different options have been evaluated:

- linear compensation using (11): the compensation angle, $\Delta \theta$ being proportional to the $q$-axis current

$\Delta \theta=a I_{q}$

- $\quad$ trigonometric compensation using (12); the compensation angle that relies on two constants, $a$ and $b$.

$\Delta \theta=\tan ^{-1}\left(\frac{a I_{q}}{b}\right)$

Compensation constants are experimentally approximated. Fig. 10b shows the position error using these two compensation methods, both leading to similar results (i.e. error $<0.1 \mathrm{rad}$ ).

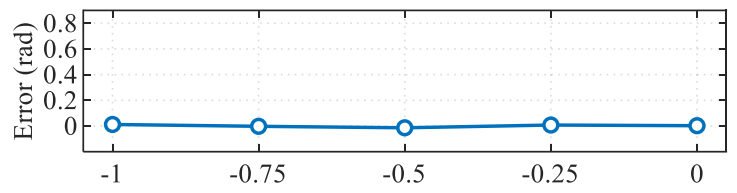

a) $I_{d}(p u)$

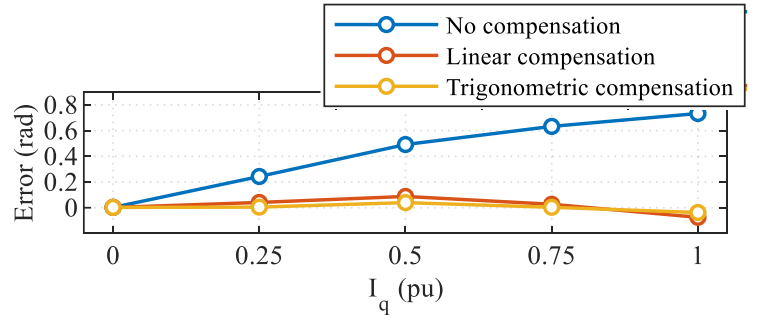

b)

Fig. 10.-. Angular position error a) when Id is varied (flux weakening), b) when Iq is varied (torque current), with and without compensation. $\omega_{\text {source }}=$ $2 \cdot \pi \cdot 10000 \mathrm{rad} / \mathrm{s}, V_{\text {source }}=10 \mathrm{~V}, \omega_{r}=200 \mathrm{rpm}$.

Performance of the proposed HER during transient operation has been also analyzed. Fig. 11 shows the experimental results when a $d$-axis current step of $-0.5 \mathrm{pu}$ is applied at $\mathrm{t}=0.35 \mathrm{~s}$ with the machine operating at constant speed. Fig. 11a shows the measured $\mathrm{d}$ and $q$-axis currents. Fig. 11b shows the rotor position measured with the proposed system without and with compensation of $q$-axis current effects as well as the rotor position measured with the encoder. It is noted that the error does not increase due to transients in the $d$-axis current.

Similarly, Fig. 12 shows the case when a $q$-axis current step of $-0.5 \mathrm{pu}$ is applied (at $\mathrm{t}=0.35 \mathrm{~s}$ for the case of without and with linear compensation, in the second case the error remaining $<0.1 \mathrm{rad}$. 
a)

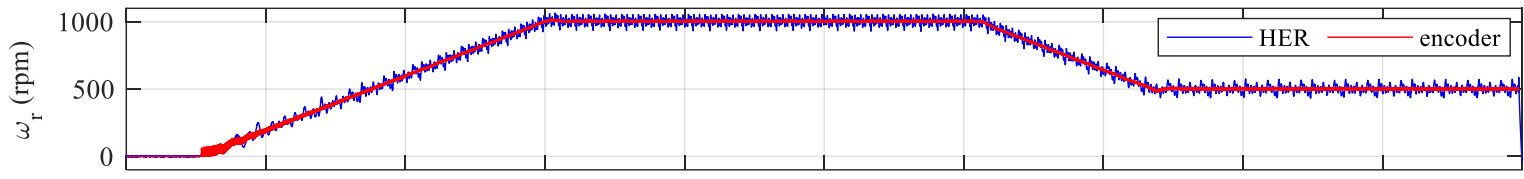

b)

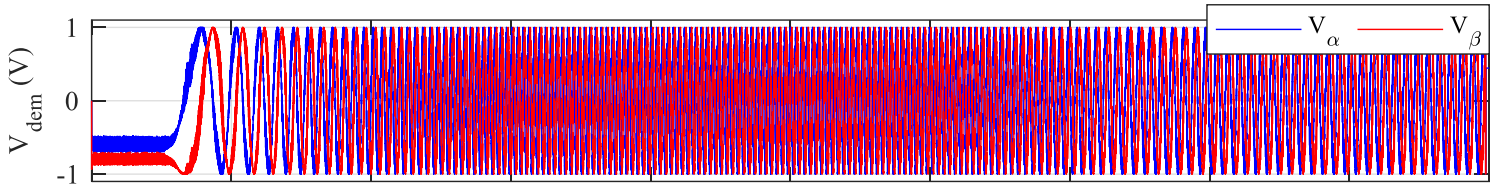

c)

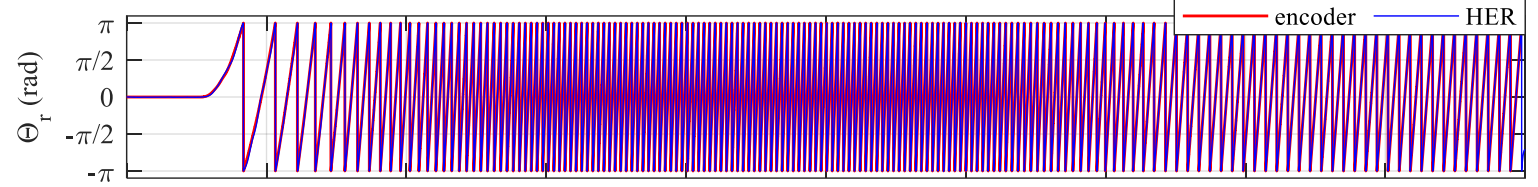

d)

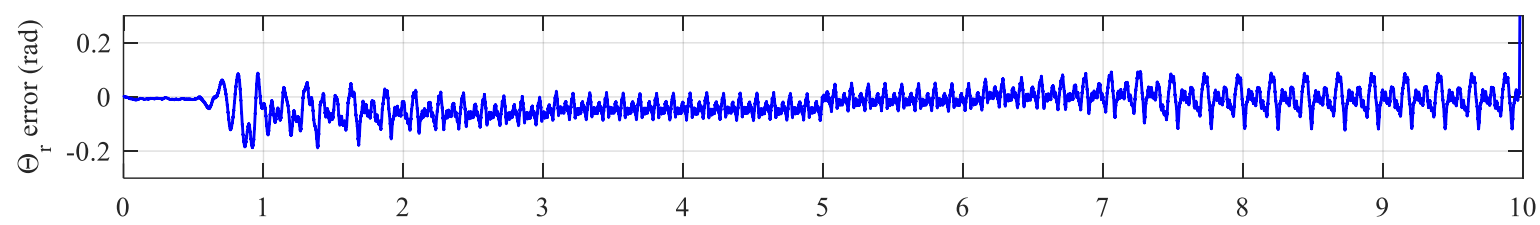

Fig. 13. Response to a speed ramp a) estimated and measured speed, b) measured leakage flux after demodulation, c) estimated position and d) estimated position error. $\omega_{\text {source }}=2 \cdot \pi \cdot 10000 \mathrm{rad} / \mathrm{s}, V_{\text {source }}=10 \mathrm{~V}$.

a)
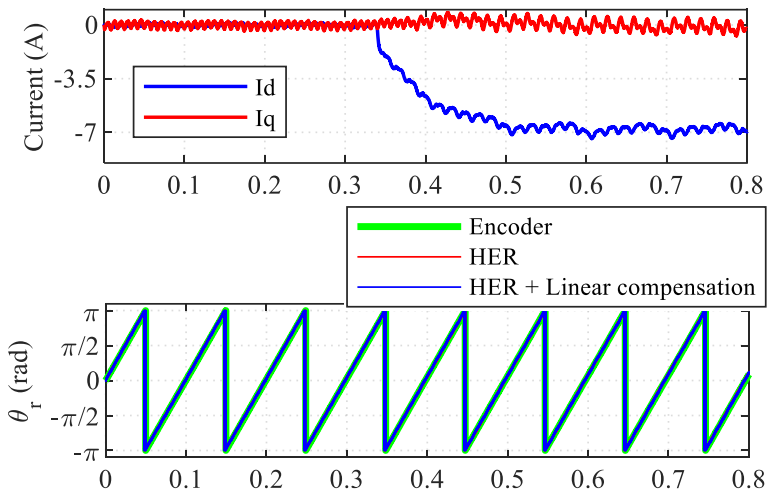

b)

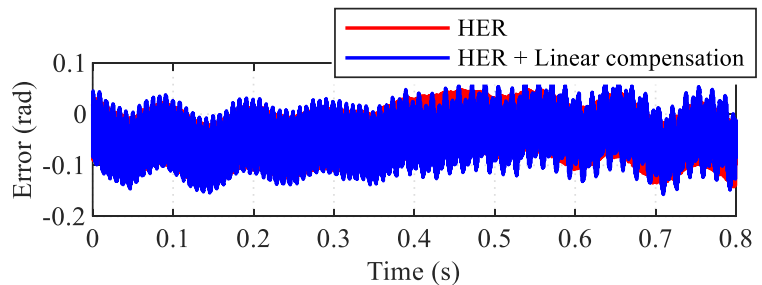

Fig. 11. Response to a $d$-axis current step a) $d$ - and $q$-axis current, b) measured position using the HER without and with $q$-axis current compensation, as from the encoder, and c) estimated position error. $\omega_{\text {source }}=$ $2 \cdot \pi \cdot 10000 \mathrm{rad} / \mathrm{s}, V_{\text {source }}=10 \mathrm{~V}, \omega_{r}=200 \mathrm{rpm}$.

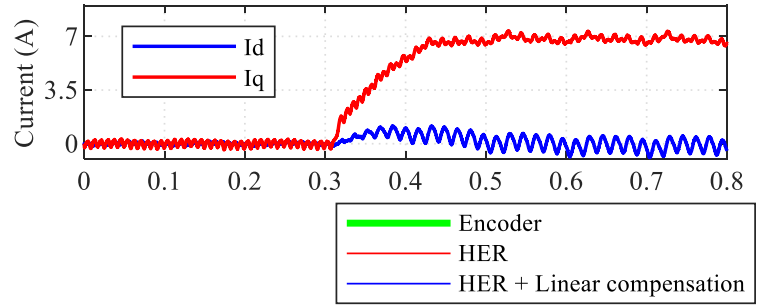

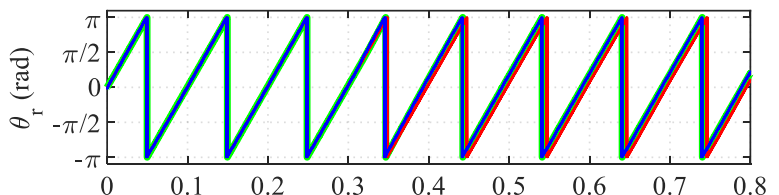

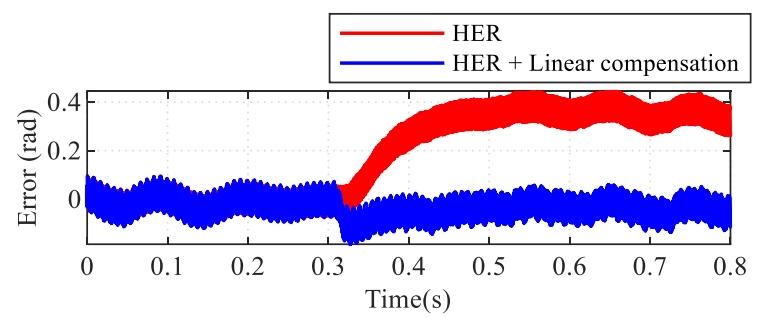

Fig. 12. Response to a $q$-axis current step a) $d$ - and $q$-axis current, b) estimated position using the HER without and with $q$-axis current compensation. Position provided by the encoder is also shown, and c) estimation error. $\omega_{\text {source }}=2 \cdot \pi \cdot 10000 \mathrm{rad} / \mathrm{s}, V_{\text {source }}=10 \mathrm{~V}, \omega_{r}=200 \mathrm{rpm}$.

\section{B. Operation at variable speed}

Performance of the HER is expected to be independent of speed. Fig. 13 shows the sensor signals and the estimated speed and position when the speed varies as shown in Fig. 13a. Results in Fig. 13d show a maximum position error of $\pm 0.05 \mathrm{rad}$ in steady state, which slightly increases during transients. Maximum error of commercially available variable reluctance resolvers ranges from \pm 0.01 rad for fourpole variable resolvers to $\pm 0.018 \mathrm{rad}$ for two-pole variable resolvers. Maximum error of commercially available brushless resolvers is in the range of $\pm 0.006 \mathrm{rad}$ 


\section{Conclusions}

This paper proposes a method to emulate a resolver using low cost Hall-effect sensors. The proposed HER device consists of two Hall-effect sensors, mounted on the end shield of the machine, 90 electrical degrees phase shifted, which measure the magnetic flux leakage of the PMSM..

Compared to commercial resolvers, the proposed HER sensor has higher error, but in turn it provides several advantages:

- Very low cost

- $\quad$ Reduced size, which eases its integration in the drive, its impact on system inertia being negligible.

- Increased robustness, as there are no moving parts or coupling.

- Lower power consumption than conventional resolvers and full compatibility with resolver circuitry.

Experimental results have been provided to demonstrate the viability of the proposed HER system.

\section{References}

[1] R. Nalepa and T. Orlowska-Kowalska, "Optimum trajectory control of the current vector of a non-salient pole PMSM in the field-weakening region," IEEE Trans. Ind. Electron., vol. 59, no. 7, pp. 2867-2876, Jul. 2012.

[2] Duane C. Hanselman, "Brushless Permanent Magnet Motor Design" New York: Mc-Graw Hill, 1994.

[3] Maxonmotorusa.com, "maxon sensor - Key information," Mar. 23, 2017. [Online]. Available: http://www.maxonmotorusa.com/

[4] Siemens.com, "Three-phase synchronous motors based on permanent magnet technology," Catalog D 86.2 • 2007, Mar. 23, 2017. [Online]. Available: http://w3.siemens.com/mcms/mc-solutions/en/motors/

[5] Technosoft.com, "Drive solutions for motion control applications," Product Overview, Mar. 23, 2017. [Online]. Available: http://www.technosoftmotion.com/en/products

[6] Abbmotion.com, "Intelligent Servo Drives and Intelligent Motors," MotiFelex e180 motion control, Mar. 23, 2017. [Online]. Available: http://www.abbmotion.com/products/servodrives/overview.asp

[7] Y. Netzer, "Linear electric encoder with facing transmitter and receiver," US Patent US2004/02525032A1, Dec. 16, 2004 iSEP:

[8] P. Kejik, S. Reymond, and R. S. Popovic, "Purely CMOS angular position sensor based on a new hall microchip," in Proc. IEEE Ind. Electron., Orlando, FL, Nov. 10-13, 2008, pp. 1777-1781. is sep?

[9] P. Kejik, S. Reymond, and R. S. Popovic, "Circular hall transducer for angular position sensing," in Proc. Transducers Eurosensors XXI Conf., Lyon, France, pp. 2593-2596, Jun. 10-14, 2007.

[10] C.S. Anoop, B. George and J. Kumar V, “Tunneling Magneto-Resistor based Angle Transducer," in Proc. Fifth International Conf. on Sensing Technology (ICST), Palmerston North, NewZeeland, pp. 431-435, Nov. 28 -Dec. 1, 2011.

[11] A. Murray, B. Hare, and A. Hirao, "Resolver position sensing system with integrated fault detection for automotive applications," in Proc. IEEE Sensors, vol. 2, pp. 864-869, Jun. 2002.

[12] X. Yongxiang, Z. Dianchen, W. Yanyu, Z. Jibin and Shang Jing, "DSP Based All-Digital Resolver-to-Digital Conversion Using DSRF-PLL ," IEEE PEMC, pp. 1210-12-15, June 2012.

[13] C.-S. Jin, I.-S. Jang, J.-N. Bae, J. Lee and W.-H. Kim, "Proposal of Improved Winding Method for VR Resolver," IEEE Trans. on Mag., 51(3): 8102404, March 2015

[14] L. Sun, "Analysis and improvement on the structure of variable reluctance resolvers," IEEE Trans. Magn., 44(8): 2002-2008, Aug. 2008.

[15] K.-C. Kim, C. S. Jin, and J. Lee, "Magnetic shield design between interior permanent magnet synchronous motor and sensor for hybrid electric vehicle,” IEEE Trans. Magn., 45(6): 2835-2838, Jun. 2009.
[16] J. Figueiredo, "Resolver models for manufacturing," IEEE Trans. Ind. Electron., 58(8): 3693-3700, Aug. 2011.

[17] L. Pecly, R. Schindeler, D. Cleveland and K. Hashtrudi-Zaad, "HighPrecision Resolver-to-Velocity Converter," IEEE Trans. on Instr. and Meas., 66(11): 2917-2928, Nov. 2017.

[18] M. Benammar and A. S. P. Gonzales, "A novel resolver converter based on a modified tracking method," in Proc. 10th IEEE Int. Conf. Netw., Sens. Control, Évry, France, pp. 586-590, Apr. 2013.

[19] R. Hoseinnezhad, A. Bab-Hadiashar, and P. Harding, "Calibration of resolver sensors in electromechanical braking systems: A modified recursive weighted least-squares approach," IEEE Trans. Ind. Electron., 54(2):1052-1060, Apr. 2007.

[20] L. Z. Sun, J. B. Zou, and Y. P. Lu, "New variable-reluctance resolver for rotor-position sensing," in Proc. IEEE Region 10th Conf. TENCON, vol. 4. Chiang Mai, Thailand, pp. 5-8, Nov. 2004.

[21] "Design-Oriented Modelling of Axial-Flux Variable-Reluctance Resolver Based on Magnetic Equivalent Circuits and Schwarz-Christoffel Mapping," IEEE Trans on Ind. Appl., 65(5): 4322-4330, May 2018.

[22] C. Attaianese and G. Tomasso, "Position Measurement in Industrial Drives by Means of Low-Cost Resolver-to-Digital Converter," IEEE Trans. on Instr. And Meas., 56(6): 2155-2159, Dec. 2007.

[23] B. P. Reddy, A. Murali and G. Shaga, "Low cost planar coil structure for inductive sensors to measure absolute angular position," 2017 2nd International Conference on Frontiers of Sensors Technologies (ICFST), Shenzhen, 2017, pp. 14-18. doi: 10.1109/ICFST.2017.8210463

[24] H. Pu, H. Wang, X. Liu, Z. Yu and K. Peng, "A High-Precision Absolute Angular Position Sensor With Vernier Capacitive Arrays Based on Time Grating," in IEEE Sensors Journal, vol. 19, no. 19, pp. 8626-8634, 1 Oct.1, 2019. doi: 10.1109/JSEN.2019.2921479

[25] M. Gasulla, Xiujun Li, G. C. M. Meijer, L. van der Ham and J. W. Spronck, "A contactless capacitive angular-position sensor," SENSORS, 2002 IEEE, Orlando, FL, USA, 2002, pp. 880-884 vol.2. doi: 10.1109/ICSENS.2002.1037224

[26] P. Kejik, S. Reymond and R. S. Popovic, "Circular Hall Transducer for Angular Position Sensing," TRANSDUCERS 2007 - 2007 International Solid-State Sensors, Actuators and Microsystems Conference, Lyon, 2007, pp. 2593-2596. doi: 10.1109/SENSOR.2007.4300702

[27] K. Bienczyk, "Angle measurement using a miniature hall effect position sensor," 2009 2nd International Students Conference on Electrodynamic and Mechatronics, Silesia, 2009, pp. 21-22. doi: 10.1109/ISCON.2009.5156096

[28] D. Fernandez, D. F. Laborda, M. Martinez, D. Reigosa, Alberto B. Diez and F. Briz, "Resolver Emulation for PMSMs Using Low Cost HallEffect Sensors" IEEE ECCE 2019, Baltimore, MD, pp: 5689-5693, 29 Sept-3 Oct., 2019.

[29] D. Fernandez, D. Hyun, Y. Park, D. Reigosa, S. B. Lee, D. M. Lee, F. Briz, "Permanent Magnet Temperature Estimation in PM Synchronous Motors Using Low Cost Hall Effect Sensors", IEEE Trans. on Industry Applications, 53(5): 4515-4525, Sept./Oct. 2017.

[30] Y. Park, D. Fernandez, S. B. Lee, D. Hyun, M. Jeong, S. K. Kommuri, C. Cho, D. Reigosa and F. Briz "On-Line Detection of Rotor Eccentricity for PMSMs based on Hall-Effect Field Sensor Measurements" IEEE Trans. on Ind. Appl., 55(2): accepted, publication pending. March/Apr. 2019.

[31] ChenYang-SensorsandMeasurements,"SelectionGuideofHallEffect Sensor Elements/ICs," CYSJ106C, Jan. 2, 2019. [Online]. Available: http://www.hallsensors.de

[32] F. Janabi-Sharifi, V. Hayward, and C.-S. J. Chen, "Discrete-time adaptive windowing for velocity estimation," IEEE Trans. Control Syst. Technol., vol. 8, no. 6, pp. 1003-1009, Nov. 2000.

[33] F. Yiyong, B. Zhuo, and C. Pengfei, "Simulation on the angular velocity and displacement measuring system based on DSRF-PLL," in Proc. IEEE Int. Conf. Syst. Sci. Eng. (ICSSE), Jul. 2014, pp. 221-226.

[34] Faulhaber.com, "Brushless DC servomotors," Series 22...BX432 3268...BX4, Jan. 9, 2019. [Online]. Available: https://fmcc.faulhaber.com

[35] http://www.allegromicro.com/en/Products/Magnetic-Linear-AndAngular-Position-Sensor-ICs/Linear-Position-Sensor-ICs/A1301-2.aspx

[36] ABB motors, "Low-voltage three-phase induction motor, M2QA225M2A", Jan. 9, 2019. [Online]. Available: https://www.abb.com/product

[37] Analog Devices, "AD2S1210, 10-Bit to16-Bit Resolver-to-Digital Converter", Jan. 9, 2019. [Online]. Available: www.analog.com 
[38] J. Bergas-Jané, C. Ferrater-Simón, G. Gross, R. Ramírez-Pisco, S. Galceran-Arellano and J. Rull-Duran, "High-Accuracy All-Digital Resolver-to-Digital Conversion," in IEEE Transactions on Industrial Electronics, vol. 59, no. 1, pp. 326-333, Jan. 2012. doi: 10.1109/TIE.2011.2143370 